\title{
Role of BCAR4 in prostate cancer cell autophagy
}

\author{
Zhiping Cai ${ }^{1 \#}$, Yapei $\mathrm{Wu}^{2 \#}$, Guanqun Ju' ${ }^{1}$, Gangmin Wang ${ }^{2}$, Bing Liu ${ }^{1}$ \\ ${ }^{1}$ Department of Urology, Changzheng Hospital, Naval Medical University, Shanghai, China; ${ }^{2}$ Department of Urology, Huashan Hospital, Fudan \\ University, Shanghai, China \\ Contributions: (I) Conception and design: Z Cai; (II) Administrative support: Z Cai; (III) Provision of study materials or patients: Z Cai; (IV) \\ Collection and assembly of data: All authors; (V) Data analysis and interpretation: Z Cai; (VI) Manuscript writing: All authors; (VII) Final approval of \\ manuscript: All authors. \\ "These authors contributed equally to this work. \\ Correspondence to: Bing Liu. Department of Urology, Changzheng Hospital, Naval Medical University, 415 Fengyang Road, Shanghai 200003, China. \\ Email: saienpapa@hotmail.com; Gangmin Wang. Department of Urology, Huashan Hospital, Fudan University, 10 Mid Wulumuqi Road, Shanghai \\ 200010, China. Email: ccy842@yeah.net.
}

Background: Increased autophagy of prostate cancer (PC) cells contributes to their resistance to chemotherapy. Recently, we reported that a long non-coding RNA (lncRNA)-breast-cancer anti-estrogen resistance 4 (BCAR4) - is highly expressed in PC and contributes to castration resistance through activation of GLI2 signaling. However, the role of BCAR4 in the regulation of PC cell autophagy is unknown and is the subject of the current study.

Methods: BCAR4 and Beclin-1 levels and the alteration in autophagy pathway genes were assessed in PC using a public database and in our own clinical specimens. The correlation between BCAR4 and Beclin-1 levels in PC and PC cell lines was determined and their regulatory relationship was assessed by overexpression and knockout assay. The final effect on autophagy was measured by microtubule-associated protein 1A/1B-light chain 3 (LC3) levels. The mechanism that underlies the control of Beclin-1 by BCAR4 was analyzed by cancer database and gain-of-function and loss-of-function approaches.

Results: BCAR4 and Beclin-1 were both upregulated in PC and were positively correlated. BCAR4 directly activated Beclin-1 at transcriptional level, which subsequently increased the ratio of LC3 II to LC3I to augment PC cell autophagy. Beclin-1 did not control levels of BCAR4. Mechanically, BCAR4 and Beclin-1 shared several targeting microRNAs, among which miR-15 and miR-146 appeared to be the mediators of the effects of BACR4 on Beclin-1.

Conclusions: BCAR4 may enhance PC cell autophagy through altering miRNA-regulated Beclin-1 expression in PC.

Keywords: Long noncoding RNA (lncRNA); breast-cancer anti-estrogen resistance 4 (BCAR4); autophagy; prostate cancer (PC); microRNAs

Submitted Sep 22, 2021. Accepted for publication Nov 04, 2021.

doi: $10.21037 /$ tau-21-929

View this article at: https://dx.doi.org/10.21037/tau-21-929

\section{Introduction}

Prostate cancer (PC) is a prevalent cancer in aged men (1). Autophagy is a catabolic pathway widely used by organisms to degrade, recycle, and reuse biological cellular compartments to improve cell survival, especially in harsh environments (2). Microtubule-associated protein 1A/1B-light chain 3 (LC3) is a soluble cellular protein, while its cytosolic form (LC3-I) forms membrane-bound LC3-phosphatidylethanolamine conjugate (LC3-II) to generate autophagosomes that engulf cytoplasmic components to fulfill the autophagy. Thus, levels of autophagic activity are often quantified by the ratio of LC3-II to LC3-I (2-4). Autophagy-associated protein 6 (Atg6, or Beclin-1) is the most important autophagyassociated protein that coordinates the occurrence of 
autophagy (5). Importantly, many cancer cells use autophagy to improve their survival at rest or when they are exposed to a radiotherapy or chemotherapy to antagonize apoptotic cell death. Thus, it is important to understand the regulation of PC cell autophagy.

There are two categories of non-coding RNAs (ncRNAs): small non-coding RNA (small molecular RNA, also known as microRNAs), and long non-coding RNA (lncRNA) (6). Although lncRNAs typically do not translate into proteins, they have many biological functions such as interaction with miRNAs or mRNAs to regulate gene expression (6). In the past, some lncRNAs have been shown to play roles in the carcinogenesis of PC $(7,8)$. We recently showed that breast-cancer anti-estrogen resistance 4 (BCAR4), a critical lncRNA functioning in tamoxifen-resistant breast cancer, activates GLI2 signaling in PC to contribute to castration resistance (9). However, the role of BCAR4 in the regulation of $\mathrm{PC}$ cell autophagy is unknown and is the subject of the current study.

We found that BCAR4 and Beclin-1 were both upregulated in PC and were positively correlated. BCAR4 directly activated Beclin-1 at transcriptional level, which subsequently increased the ratio of LC3 II to LC3I to augment PC cell autophagy. Beclin-1 did not control levels of BCAR4. Mechanically, BCAR4 and Beclin-1 shared several targeting microRNAs, among which miR-15 and miR-146 appeared to be the mediators of the effects of BACR4 on Beclin-1.

We present the following article in accordance with the MDAR reporting checklist (available at https://dx.doi. org/10.21037/tau-21-929).

\section{Methods}

\section{Protocols and patient specimens}

All procedures performed in this study involving human participants were in accordance with the Declaration of Helsinki (as revised in 2013). The study was approved by the Ethics Committee of the Shanghai Changzheng Hospital (JS-254). Use of specimens was approved by donors (no number) who returned signed informed consent.

We evaluated ninety PC tissues with matched adjacent normal prostate tissues (NT) without prior chemotherapy or radiotherapy in male patients aged from 45 to 78 years. Samples were histologically diagnosed independently by two experienced pathologists and extracted RNA was kept in $-70{ }^{\circ} \mathrm{C}$ before proceeding. Proper randomization and blinding method were used, and the 5-year survival of patients was followed.

\section{Cell culture}

Human PC cell lines (PC346, LNCap, MDAPC1 2a/b, C4-2, PC3, and DU145) were obtained from the American Type Culture Collection (Catalog number CRL-2876, CRL-1740, CRL-2242, CRL-3314, CRL-1435, and HTB81, respectively, ATCC, Rockville, MD, USA). Human PC cell line (BPH1) was obtained from Accegen Biotechnology (Catalog number ABC-TC454S, Fairfield, NJ, USA). All lines were maintained in DMEM (Invitrogen, Rockville, MD, USA) suppled with $5 \%$ fetal bovine serum (FBS; Sigma-Aldrich, Rockville, MD, USA) in a humidified incubator at $37^{\circ} \mathrm{C}$ with a $5 \% \mathrm{CO}_{2}$ atmosphere.

\section{Plasmids and transfection}

The BCAR4 and siRNA-BCAR4 plasmids have been previously described (9). The plasmids that express recombinant Beclin-1, siRNA, or a scrambled sequence for Beclin-1 (si-Beclin-1; scrambled), or express miR-15, miR-105, miR-146, and antisense for miR-15 (asmiR-15), as-miR-105, and as-miR-146 under a CMV promoter were all purchased from GenePharma and Santa Cruz (Beijing, China). The sequence for si-BCAR4 was 5'-GGGACTTGAGTTATGTTGGTGGCTA-3' and the sequence for the scrambled sequence was 5'-TGAGTTGGCGCGACTG-3'. Scrambled shRNAs were used as negative controls. Transfection was performed with $1.5 \mu \mathrm{g}$ plasmids using Lipofectamine 3000 (Invitrogen) for 24 hours.

\section{$R N A$ extraction and quantitative real-time polymerase chain reaction ( $R T-q P C R)$}

Total RNA, including ncRNAs, was extracted with the miRNeasy Kit (Qiagen, Shanghai, China) following the manufacturer's protocol. cDNA was generated with a RT Synthesis Kit (Qiagen) which was used as the template in RT-qPCR with a commercial SYBR Green PCR Kit (Qiagen, Shanghai, China) and designed primers from Qiagen. The RT-qPCR reactions were performed in duplicate. The levels of gene expression were quantified with the $2^{-\Delta \Delta C t}$ method and presented as relative values after sequential normalization to $\alpha$-tubulin and the experimental controls. 

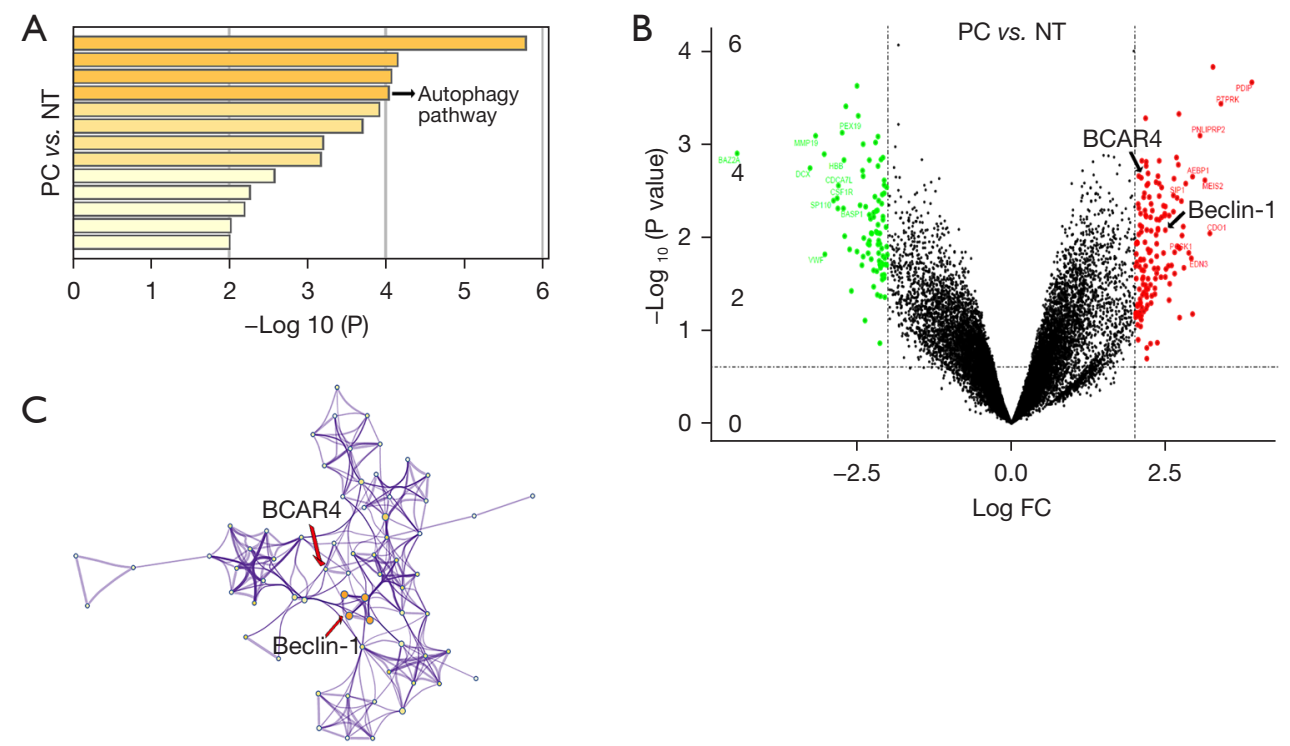

Figure 1 Analysis of public databases shows enhanced autophagy pathway and Beclin-1 levels in PC. PC data from public databases were obtained and the online tool GEO2R was used to screen associated genes and pathways. (A) Pathway enrichment analysis were assessed by the Metascape online tool, showing that the enriched pathways included autophagy; (B) a volcano map to show that both BACR4 and Beclin-1 were upregulated in PC; (C) protein-protein interaction network demonstrated a close, although not direct, interaction between BACR4 and Beclin-1. BCAR4, breast-cancer anti-estrogen resistance 4; PC, prostate cancer.

\section{Western blot}

The protein was obtained through RIPA buffer containing protease and phosphatase inhibitors (cOmplete ULTRA Tablets, Roche, Nutley, NJ, USA) mediated cell lysis, and the protein quantification was performed using NanoDrop microvolume spectrophotometers. The proteins were next subjected to an SDS-PAGE and then transferred to nitrocellulose membranes. After blocking with 5\% nonfat milk, the membranes were probed with rabbit anti-LC3, rabbit-anti-Beclin-1, or rabbit-anti- $\alpha$-tubulin (Catalog number 4599, 3495 and 5335, respectively, Cell Signaling Technology, Danvers, MA, USA). Secondary antibodies used HRPconjugated antibodies against rabbit (Jackson ImmunoResearch Labs, West Grove, PA, USA). Protein levels were presented as relative values after sequential normalization to $\alpha$-tubulin, and the experimental controls and densitometry of Western blots was quantified with NIH ImageJ software.

\section{Bioinformatics and statistical analysis}

The public database from the Gene Expression Omnibus (GEO) was used in the current study (10). Several gene expression profiles in PC (GSE161304, GSE134171, GSE134170, GSE134168, and GSE134073) were used for GEO2R online analysis for detecting the differentially expressed genes (DEGs), after which the $\mathrm{P}$ value, adjusted $\mathrm{P}$ value, and $\log \mathrm{FC}$ were calculated. Pathway enrichment analyses of DEGs were performed at Metascape (11), and the data were statistically analyzed with GraphPad Prism 6 (GraphPad, Chicago, IL, USA). Student's $\mathrm{T}$ test was performed to compare the data of two groups and the values were expressed as mean \pm standard deviation (SD). When $\mathrm{P}<0.05$, the data was considered as significant. Bivariate correlations were calculated by Spearman's Rank Correlation Coefficients, and Kaplan-Meier curve was applied to record the overall survival of included patients. Experiments were repeated 3-4 times.

\section{Results}

\section{Analysis of a public database shows enbanced autophagy pathway and Beclin-1 levels in PC}

To evaluate the role of BCAR4 in PC autophagy, we first obtained data from a public database and used the online tool GEO2R to screen DEGs between PC and adjacent NT. Pathway enrichment analysis was analyzed using the Metascape online tool and showed autophagy pathway as a major altered pathway in PC (Figure 1A). Among 

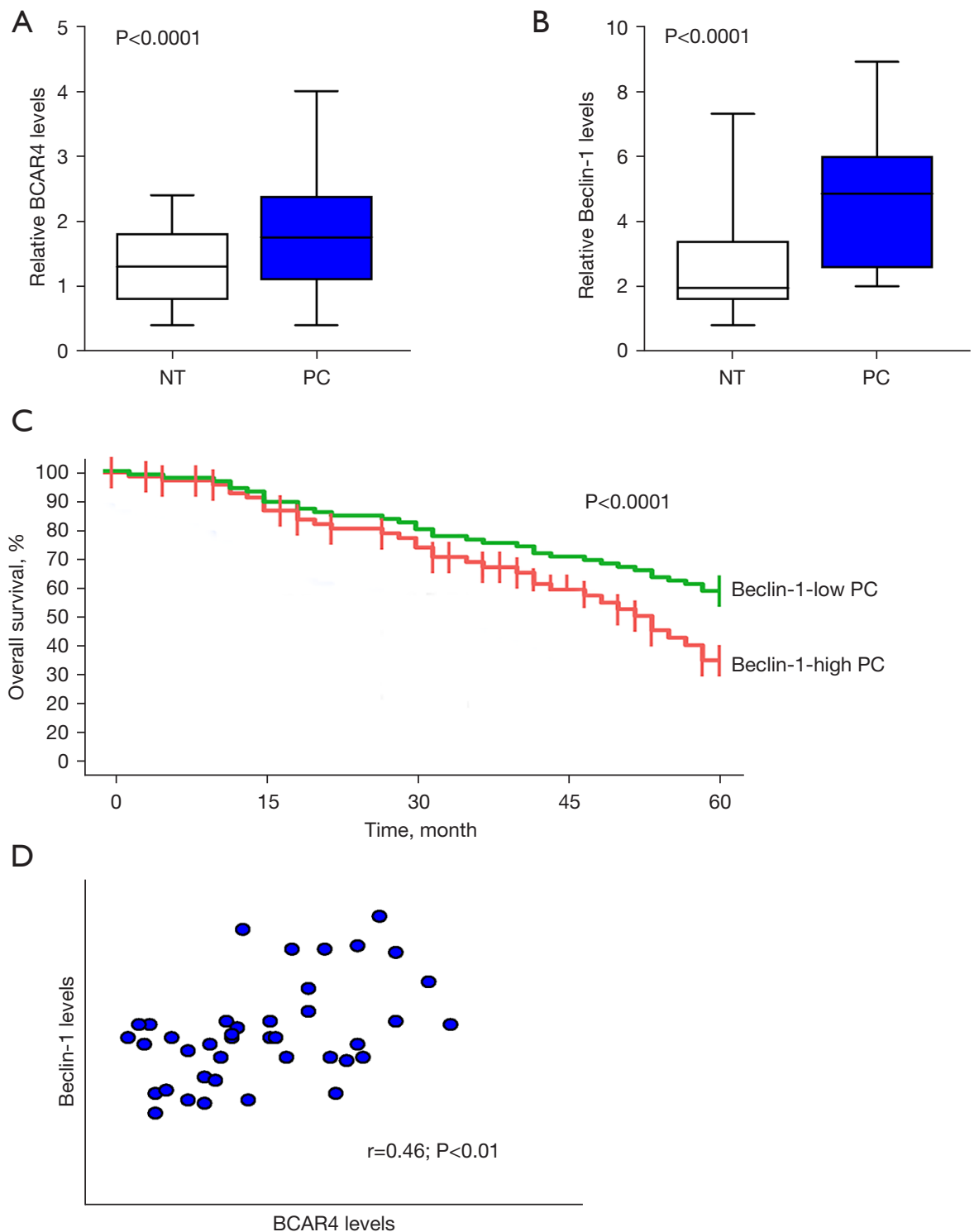

Figure 2 Analysis of our clinic samples shows a positive correlation between BACR4 and Beclin-1/autophagy in PC. (A,B) BCAR4 (A) and Beclin-1 (B) levels were examined in PC and paired adjacent normal prostate tissues (NT) by RT-qPCR. (C) The PC tissues were classified into two groups (Beclin-1-low and Beclin-1-high) using the median value of Beclin-1 in all cases as the cutoff point. A Kaplan-Meier curve showed patients' overall survival. (D) A correlation test for BACR4 and Beclin-1 levels in PC specimens. N=50. BCAR4, breast-cancer antiestrogen resistance 4; PC, prostate cancer.

all DEGs, significantly downregulated and upregulated genes were detected, and interestingly, both BACR4 and Beclin-1 were found significantly upregulated in PC (Figure 1B). Moreover, protein-protein interaction network demonstrated a close, although not direct, interaction between BACR4 and Beclin-1 (Figure 1C). These data suggest a possible relationship between BACR4 and Beclin-1/autophagy in PC.

\section{Analysis of clinic samples shows a positive correlation between BACR4 and Beclin-1/autophagy in PC}

To further provide evidence for a possible relationship between BACR4 and Beclin-1/autophagy in PC, we analyzed our clinic samples as previously described (9), and found significant increases in both BACR4 (Figure 2A) and Beclin-1 (Figure 2B) in PC, compared to NT. The PC 


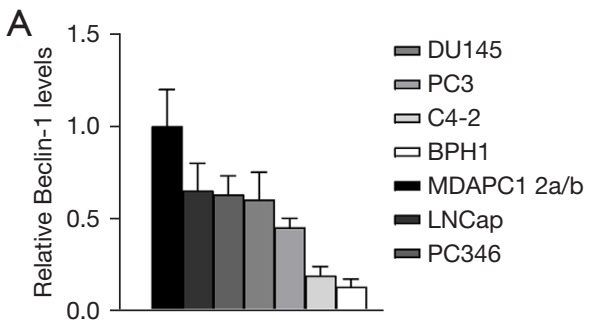

B
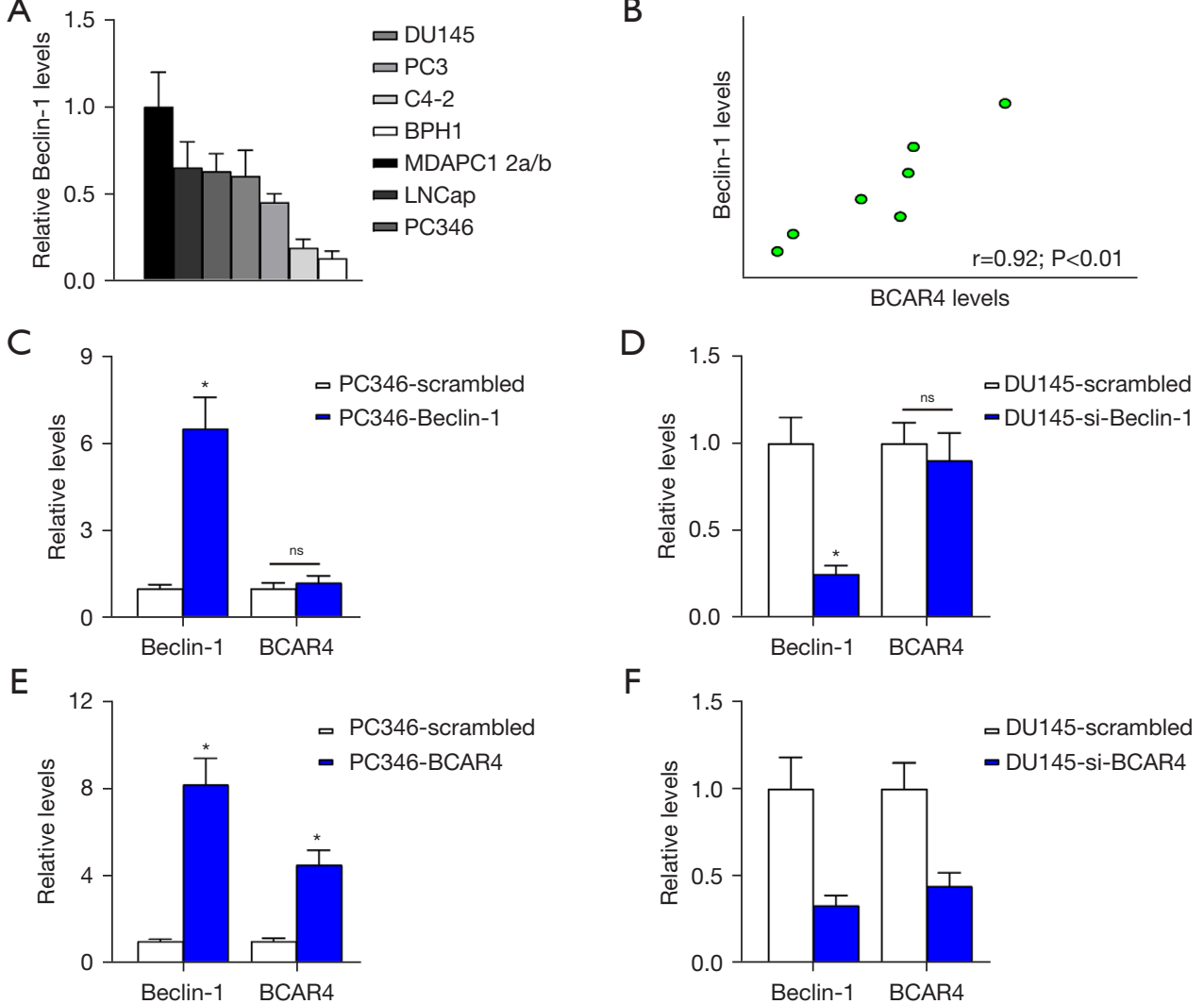

Figure 3 BCAR4 regulates Beclin-1 expression in PC cells. (A) RT-qPCR for Beclin-1 levels in seven commonly used PC cell lines. (B) A correlation test for BACR4 and Beclin-1 levels in the seven PC cell lines. (C,D) Low-Beclin-1-expressing PC346 cells were transfected with either scrambled or Beclin-1 plasmids, while high-Beclin-1-expressing DU145 cells were transfected with either scrambled or siBeclin-1 plasmids. RT-qPCR for Beclin-1 and BCAR4 levels in transfected PC346 cells (C) and in transfected DU145 cells (D). (E,F) Low-BCAR4-expressing PC346 cells were transfected with either scrambled or BCAR4 plasmids, while high-BCAR4-expressing DU145 cells were transfected with either scrambled or si-BCAR4 plasmids. RT-qPCR for BCAR4 and Beclin-1 levels in transfected PC346 cells (E) and in transfected DU145 cells (F). *, $\mathrm{P}<0.05$. NS, non-significant. N=5. BCAR4, breast-cancer anti-estrogen resistance 4; PC, prostate cancer.

specimens were grouped into a Beclin-1-high and Beclin-1low group, respectively, using the median value of all cases as the cutoff point, and the results showed overall survival was significantly better in those with low Beclin-1 levels, shown in a Kaplan-Meier curve $(\mathrm{P}<0.0001$, Figure $2 C)$. Moreover, the BACR4 and Beclin-1 level in each individual patient exhibited a strong positive correlation $(\mathrm{r}=0.46 ; \mathrm{P}<0.01$; Figure 2D). These data suggest a regulatory relationship between BACR4 and Beclin-1/autophagy in PC.

\section{BCAR4 regulates Beclin-1 expression in PC cells}

We then tried to prove this relationship in PC cells. First, we examined seven commonly used PC cell lines and found that PC346 expressed the least level of Beclin-1, while DU145 expressed the highest level (Figure 3A). Interestingly, we have previously shown that PC346 expressed the least level of BCAR4, while DU145 expressed the highest level of BCAR4 (9). Thus, a correlation test for BACR4 and Beclin-1 levels in the seven lines was performed, and, surprisingly, showed a very strong positive correlation $(\mathrm{r}=0.92 ; \mathrm{P}<0.01$; Figure $3 B)$. We then assessed whether alteration in either BACR4 or Beclin-1 could change the levels of the other by transfecting low-Beclin1-expressing PC346 cells with either scrambled or Beclin-1 plasmids, while high-Beclin-1-expressing DU145 cells were 

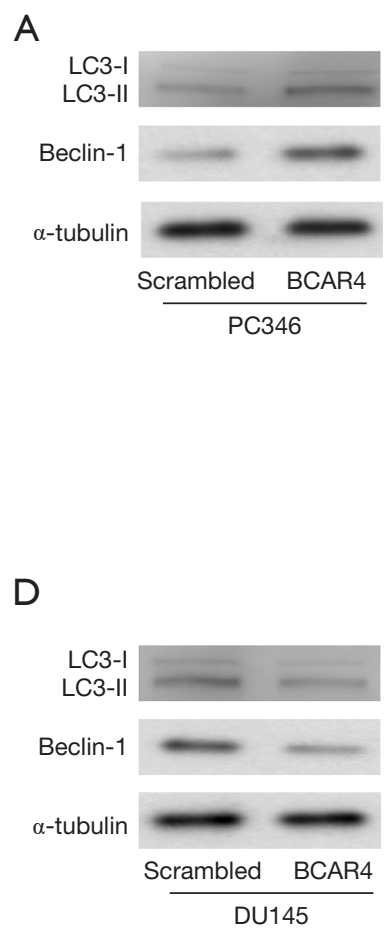

B

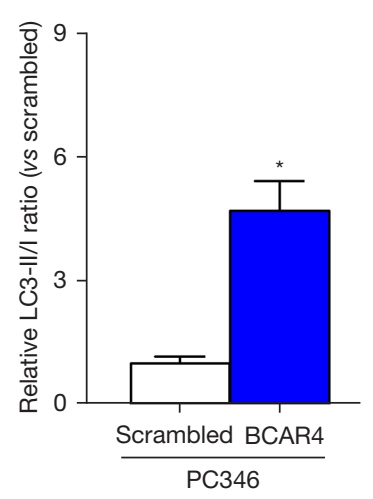

E

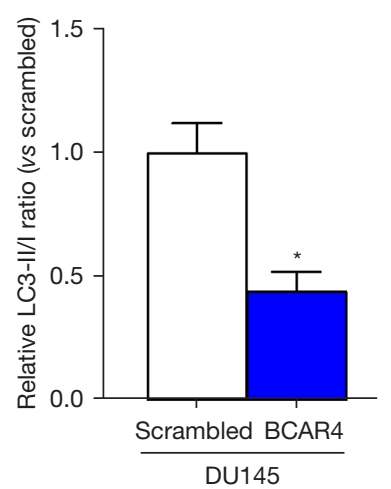

C

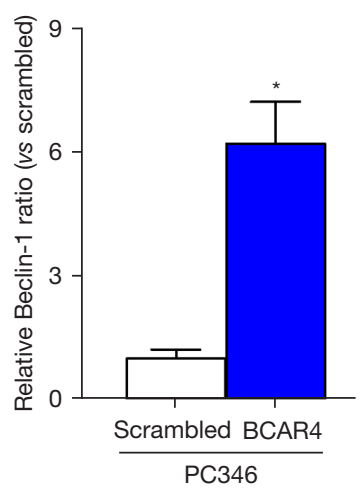

$\mathrm{F}$

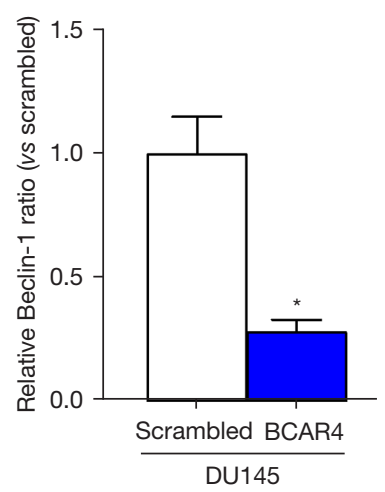

Figure 4 Regulation of Beclin-1 by BCAR4 alters PC cell autophagy. (A,B) Immunoblots for autophagy-associated genes LC3 and Beclin-1 in transfected PC346 cells, shown by representative blots (A), and by quantification for ratio LC3-II vs. I (B) and Beclin-1 (C). (D-F) Immunoblots for LC3 and Beclin-1 in transfected DU145 cells, shown by representative blots (D), and by quantification for ratio LC3-II vs. $\mathrm{I}(\mathrm{E})$ and Beclin-1 (F). *, $\mathrm{P}<0.05 . \mathrm{N}=5$. BCAR4, breast-cancer anti-estrogen resistance 4; PC, prostate cancer; LC3, light chain 3.

transfected with either scrambled or si-Beclin-1 plasmids. The results showed that transfection with Beclin-1 significantly increased Beclin-1 levels in PC346 cells without altering BCAR4 levels (Figure 3C), while transfection with si-Beclin-1 significantly decreased Beclin-1 levels in DU145 cells without altering BCAR4 levels (Figure 3D). This indicated Beclin-1 does not regulate BCAR4. In another set of experiments, low-BCAR4-expressing PC346 cells were transfected with either scrambled or BCAR4 plasmids, while high-BCAR4-expressing DU145 cells were transfected with either scrambled or si-BCAR4 plasmids. We found that transfection with BCAR4 significantly increased both BCAR4 and Beclin-1 levels in PC346 cells (Figure 3E), while transfection with si-BCAR4 significantly decreased both BCAR4 and Beclin-1 levels in DU145 cells (Figure 3F). Thus, BCAR4 appears to regulate Beclin-1 levels in PC cells.

\section{Regulation of Beclin-1 by BCAR4 alters PC cell autophagy}

Next, we analyzed whether the regulation of Beclin-1 by BCAR4 altered PC cell autophagy by analyzing the changes in Beclin-1 levels and LC3-II/I ratio as an indicator for autophagy levels in transfected cells. We found that transfection of BCAR4 in low-BCAR4-expressing PC346 cells not only significantly increased the levels of Beclin-1, but also significantly increased the levels of LC3-II/I ratio, shown by representative immunoblots (Figure $4 A$ ), and by quantification (Figure $4 B, 4 C$ ). On the other hand, transfection of si-BCAR4 in high-BCAR4-expressing DU145 cells not only significantly decreased the levels of Beclin-1, but also significantly decreased the levels of LC3II/I ratio, shown by representative immunoblots (Figure $4 D$ ), and by quantification (Figure $4 E, 4 F$ ). Together, these data suggest that the BCAR4-mediated increases in Beclin-1 

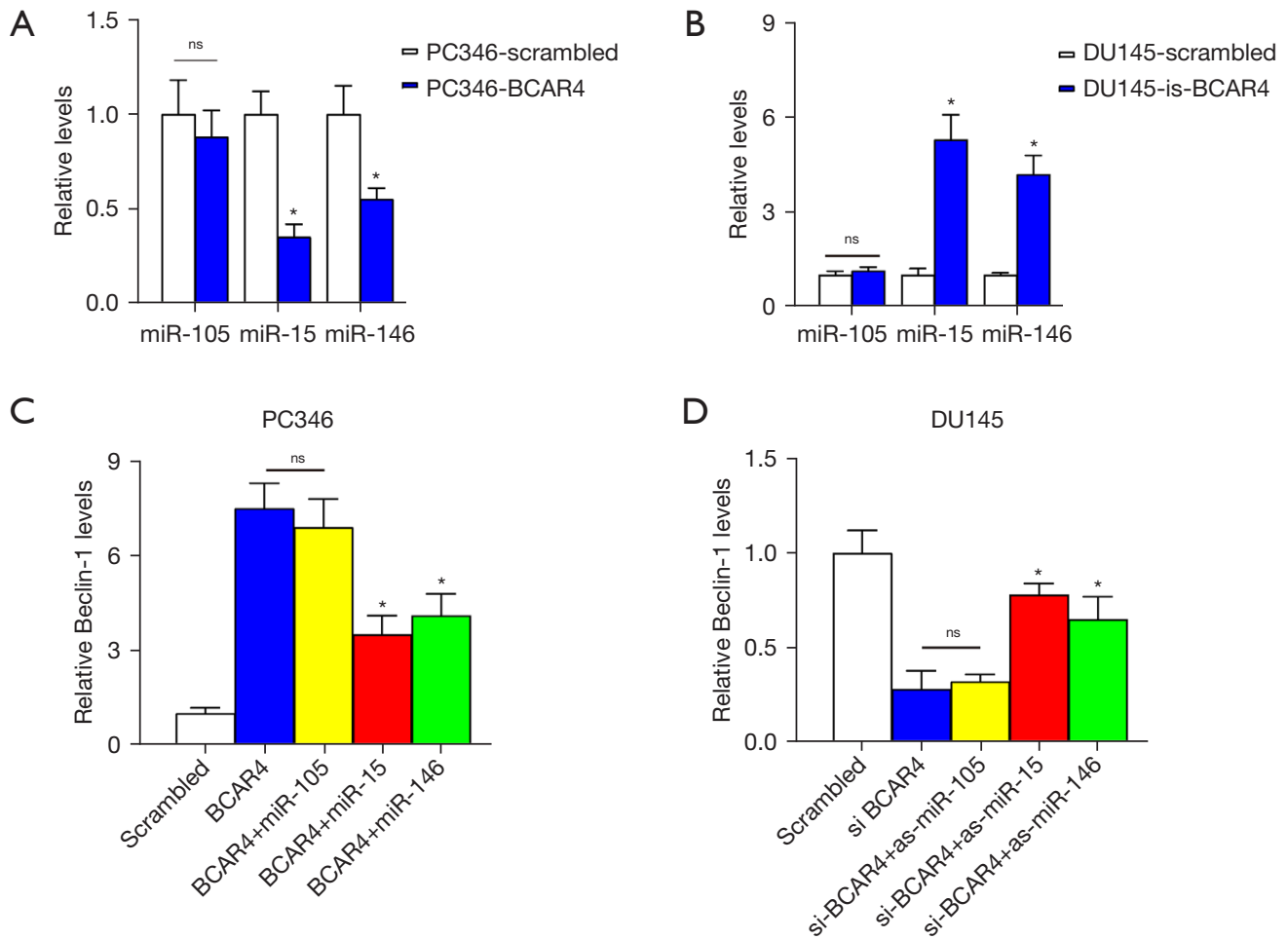

Figure 5 BCAR4 likely regulates Beclin-1 through miR-15 and miR-146 in PC cells. (A,B) RT-qPCR for the levels of miR-105, miR-15, and miR-146 in BCAR4/scrambled-transfected PC345 cells (A) and in si-BCAR4/scrambled-transfected DU145 cells (B). (C,D) RT-qPCR for the levels of Beclin-1 in PC345 cells transfected with scrambled or BCAR4 or combined BCAR4 with either miR-105, miR-15, or miR146 (C), and in DU145 cells transfected with scrambled or si-BCAR4 or combined si-BCAR4 with either as-miR-105, as-miR-15, or asmiR-146 (D). * $\mathrm{P}<0.05$. NS, non-significant. N=5. BCAR4, breast-cancer anti-estrogen resistance 4; PC, prostate cancer.

promote PC cell autophagy.

\section{BCAR4 likely regulates Beclin-1 through miR-15 and miR-146 in PC cells}

Finally, we studied the mechanisms underlying the regulation of Beclin-1 in PC by BCAR4. As a lncRNA, BCAR4 typically regulates gene expression through its interaction with miRNAs, which must have complementary sequences with both targeting lncRNA and the 3'-UTR for the mRNA of the targeting gene, here as BCAR4 and Beclin-1, respectively. Therefore, we used the online miRcode tool to predict all Beclin-1 (Table S1; 36 candidates obtained) and BCAR4 (Table S2; 53 candidates obtained) targeting miRNAs, among which nine groups were shared (miR-7, miR-105, miR138, miR-146, miR-15, miR-150, miR-17, miR-216, and miR-29). The miRCancer online tool was then used to examine the miRNAs among these nine groups that have been reported to be downregulated in PC (available online: https://cdn.amegroups.cn/static/public/tau-21929-01.pdf) (12), and miR-105, miR-15, and miR-146 were obtained as final candidates to be tested. We then tested whether levels of these three miRNAs could be altered in BCAR4/si-BCAR4-transfected PC cells and found transfection of BCAR4 in low-BCAR4-expressing PC346 cells did not significantly alter the levels of miR105 , but significantly decreased the levels of miR-15 and miR-146 (Figure 5A). On the other hand, transfection of si-BCAR4 in high-BCAR4-expressing DU145 cells did not significantly alter the levels of miR-105, but significantly increased the levels of miR-15 and miR146 (Figure 5B). Moreover, transfection with miR-15 and miR-146, but not with miR-105, significantly attenuated the effects of altered BCAR4 on Beclin-1 levels in both PC346 cells (Figure 5C) and DU145 cells (Figure 5D). These data suggest that BCAR4 likely regulates Beclin-1 through miR-15 and miR-146 in PC cells. 


\section{Discussion}

Beclin-1 is the mammalian orthologue of yeast ATG6, and plays a pivotal role in the regulation of autophagy, in which Beclin-1 has crosstalk with a number of autophagy cofactors, including ATG14L, Rubicon, Ambra1, UVRAG, nPIST, VMP1, Bif-1, SLAM, IP3R, HMGB1 and PINK, to initiate the process of autophagy (13). A previous study has demonstrated the importance of Beclin-1 in the tumorigenesis of PC by showing it likely regulates the malignant transformation of prostate tissue and promotes $\mathrm{PC}$ cell differentiation, progression, and aggressive potential (14).

Interestingly, Beclin-1 has been shown to be controlled by miRNAs $(15,16)$ in PC and by lncRNAs in other cancers (17-20). However, a role of BCAR4 in the regulation of Beclin-1 and autophagy has not been reported before now. BCAR4 is a lncRNA, which typically regulates gene expression through its interaction with miRNAs which further regulate protein translation of certain target genes through their complementary sequence binding to the 3'-UTR for the mRNA (21). Moreover, the interaction between lncRNA and miRNAs is also through complementary sequence binding. The lncRNA negatively regulates the level of the target miRNA while miRNA negatively regulates the translation of the target gene (21). Therefore, such a regulatory pair is characteristic in that the direction of the changed expression level of lncRNA, and the gene is typically the same, but different with the miRNAs (21).

Based on these criteria, we screened all candidate miRNAs and found miR-105, miR-15, and miR-146, to meet all these requirements. However, only miR-15 and miR-146 passed the gain-of-function and loss-offunction tests, suggesting they may be the mediators for the regulation of Beclin-1 by BCAR4. In contrast, MiR-105 did not seem to be involved in this regulation, which may be due to the expression level, assess to the control point, or other mechanisms. Interestingly, miR-146 has been shown to regulate cell autophagy in PC $(22,23)$, and miR15 has been reported to control PC progression through interacting with a number of genes (24). However, a direct crosstalk between Beclin-1 and miR-15/miR-146 has not been shown before.

Thus, to the best of our knowledge, our study not only showed a novel role of BCAR4 in the regulation of autophagy in PC, but also showed a possible innovative mechanism by which Beclin-1 can be regulated by miR15 and miR-146. Further studies may evaluate the possible presence of this regulatory axis in other cancers, and these approaches may help to better characterize this molecular pathway and its importance in cancer cell autophagy.

\section{Acknowledgments}

Funding: None.

\section{Footnote}

Reporting Checklist: The authors have completed the MDAR reporting checklist. Available at https://dx.doi. org/10.21037/tau-21-929

Data Sharing Statement: Available at https://dx.doi. org/10.21037/tau-21-929

Conflicts of Interest: All authors have completed the ICMJE uniform disclosure form (available at https://dx.doi. org/10.21037/tau-21-929). The authors have no conflicts of interest to declare.

Ethical Statement: The authors are accountable for all aspects of the work in ensuring that questions related to the accuracy or integrity of any part of the work are appropriately investigated and resolved. All procedures performed in this study involving human participants were in accordance with the Declaration of Helsinki (as revised in 2013). The study was approved by the Ethics Committee of the Shanghai Changzheng Hospital (JS-254). Use of specimens was approved by donors (no number) who returned signed informed consent.

Open Access Statement: This is an Open Access article distributed in accordance with the Creative Commons Attribution-NonCommercial-NoDerivs 4.0 International License (CC BY-NC-ND 4.0), which permits the noncommercial replication and distribution of the article with the strict proviso that no changes or edits are made and the original work is properly cited (including links to both the formal publication through the relevant DOI and the license). See: https://creativecommons.org/licenses/by-nc-nd/4.0/.

\section{References}

1. Fonseka LN, Kallen ME, Serrato-Guillen A, et al. Cytogenetics and Molecular Genetics of Prostate Cancer: A Comprehensive Update. J Assoc Genet Technol 
2015;41:100-11.

2. Fei J, Xu A, Zeng W, et al. The molecular basis for $\mathrm{p} 53$ inhibition of autophagy in porcine fibroblast cells. Transl Cancer Res 2019;8:876-86.

3. Guo JY, Xia B, White E. Autophagy-mediated tumor promotion. Cell 2013;155:1216-9.

4. White E. Deconvoluting the context-dependent role for autophagy in cancer. Nat Rev Cancer 2012;12:401-10.

5. Levine B, Kroemer G. Autophagy in the pathogenesis of disease. Cell 2008;132:27-42.

6. Awan HM, Shah A, Rashid F, et al. Primate-specific Long Non-coding RNAs and MicroRNAs. Genomics Proteomics Bioinformatics 2017;15:187-95.

7. Misawa A, Takayama KI, Inoue S. Long non-coding RNAs and prostate cancer. Cancer Sci 2017;108:2107-14.

8. Al Olama AA, Kote-Jarai Z, Giles GG, et al. Multiple loci on 8 q24 associated with prostate cancer susceptibility. Nat Genet 2009;41:1058-60.

9. Cai Z, Wu Y, Li Y, et al. BCAR4 activates GLI2 signaling in prostate cancer to contribute to castration resistance. Aging (Albany NY) 2018;10:3702-12.

10. Barrett T, Wilhite SE, Ledoux P, et al. NCBI GEO: archive for functional genomics data sets--update. Nucleic Acids Res 2013;41:D991-5.

11. Zhou Y, Zhou B, Pache L, et al. Metascape provides a biologist-oriented resource for the analysis of systemslevel datasets. Nat Commun 2019;10:1523.

12. Xie B, Ding Q, Han H, et al. miRCancer: a microRNAcancer association database constructed by text mining on literature. Bioinformatics 2013;29:638-44.

13. Chen L, Du H, Luo F, et al. Beclin-1 expression serves as an important biomarker for car-cinogenesis and evolution in lung adenocarcinoma presenting as ground glass opacity. Transl Cancer Res 2020;9:4204-11.

14. Baspinar S, Bircan S, Orhan H, et al. The relation of beclin 1 and bcl-2 expressions in high grade prostatic intraepithelial neoplasia and prostate adenocarcinoma: a tissue microarray study. Pathol Res Pract 2014;210:412-8.

15. Nam RK, Benatar T, Amemiya Y, et al. Mir-139 Regulates Autophagy in Prostate Cancer Cells Through
Beclin-1 and mTOR Signaling Proteins. Anticancer Res 2020;40:6649-63.

16. Xiao W, Dai B, Zhu Y, et al. Norcantharidin induces autophagy-related prostate cancer cell death through Beclin-1 upregulation by miR-129-5p suppression. Tumour Biol 2015. [Epub ahead of print]. doi: 10.1007/ s13277-015-4488-6.

17. Hu F, Tao X, Zhao L, et al. LncRNA-PVT1 aggravates severe acute pancreatitis by promoting autophagy via the miR-30a-5p/Beclin-1 axis. Am J Transl Res 2020;12:5551-62.

18. Zhang J, He JF. LncRNA-MALAT1 influences myocardial infarction by regulating miR-30a/beclin-1 pathway. Eur Rev Med Pharmacol Sci 2020;24:885-92.

19. Pu Z, Wu L, Guo Y, et al. LncRNA MEG3 contributes to adenosine-induced cytotoxicity in hepatoma HepG2 cells by downregulated ILF3 and autophagy inhibition via regulation $\mathrm{PI} 3 \mathrm{~K}-\mathrm{AKT}-\mathrm{m}$ TOR and beclin-1 signaling pathway. J Cell Biochem 2019;120:18172-85.

20. Wang K, Yang C, Shi J, et al. Ox-LDL-induced lncRNA MALAT1 promotes autophagy in human umbilical vein endothelial cells by sponging miR-216a-5p and regulating Beclin-1 expression. Eur J Pharmacol 2019;858:172338.

21. Janakiraman H, House RP, Gangaraju VK, et al. The Long (lncRNA) and Short (miRNA) of It: TGF $\beta$-Mediated Control of RNA-Binding Proteins and Noncoding RNAs. Mol Cancer Res 2018;16:567-79.

22. Gao S, Zhao Z, Wu R, et al. MiR-146b inhibits autophagy in prostate cancer by targeting the PTEN/Akt/mTOR signaling pathway. Aging (Albany NY) 2018;10:2113-21.

23. Hanwei J, Nie X, Zhu H, et al. miR-146b-5p Plays a Critical Role in the Regulation of Autophagy in $\Delta$ per Brucella melitensis-Infected RAW264.7 Cells. Biomed Res Int 2020;2020:1953242.

24. Bonci D, Coppola V, Musumeci M, et al. The miR-15amiR-16-1 cluster controls prostate cancer by targeting multiple oncogenic activities. Nat Med 2008;14:1271-7.

(English Language Editor: B. Draper)
Cite this article as: Cai Z, Wu Y, Ju G, Wang G, Liu B. Role of BCAR4 in prostate cancer cell autophagy. Transl Androl Urol 2021;10(11):4253-4261. doi: 10.21037/tau-21-929 
Supplementary

Table S1: Beclin-1-targeting miRNAs

\begin{tabular}{|c|c|c|c|c|c|c|c|}
\hline \multirow{2}{*}{ microRNA family } & \multirow{2}{*}{ Seed position } & \multirow{2}{*}{ Seed type } & \multirow{2}{*}{ Transcript region } & \multirow{2}{*}{ Repeat } & \multicolumn{3}{|c|}{ Conservation } \\
\hline & & & & & Primates & Mammals & Other vert. \\
\hline miR-551a & chr17:40966646 & 7-mer-m8 & CDS & no & $89 \%$ & $70 \%$ & $31 \%$ \\
\hline miR-7/7ab & chr17:40963678 & 7-mer-m8 & CDS & no & $89 \%$ & $83 \%$ & $8 \%$ \\
\hline miR-93/93a/105/106a/291a-3p/294/295/302abcde/372/373/428/519a/520be/520acd-3p/1378/1420ac & chr17:40962422 & 7-mer-A1 & 3pUTR & no & $56 \%$ & $0 \%$ & $0 \%$ \\
\hline miR-96/507/1271 & chr17:40970871 & 7-mer-m8 & CDS & no & $78 \%$ & $57 \%$ & $38 \%$ \\
\hline miR-96/507/1271 & chr17:40971530 & 7-mer-A1 & $5 p U T R$ & no & $78 \%$ & $35 \%$ & $0 \%$ \\
\hline miR-138/138ab & chr17:40971067 & 7-mer-A1 & $5 p$ UTR & no & $67 \%$ & $48 \%$ & $0 \%$ \\
\hline miR-139-5p & chr17:40971150 & 8-mer & $5 p U T R$ & no & $56 \%$ & $26 \%$ & $0 \%$ \\
\hline miR-141/200a & chr17:40966633 & 7-mer-m8 & CDS & no & $89 \%$ & $70 \%$ & $8 \%$ \\
\hline miR-144 & chr17:40962213 & 7-mer-m8 & $3 p U T R$ & no & $67 \%$ & $65 \%$ & $8 \%$ \\
\hline miR-144 & chr17:40963797 & 7-mer-m8 & CDS & no & $89 \%$ & $43 \%$ & $15 \%$ \\
\hline miR-145 & chr17:40975812 & 7 -mer-m8 & CDS,5pUTR & no & $89 \%$ & $74 \%$ & $31 \%$ \\
\hline miR-146ac/146b-5p & chr17:40971236 & 8-mer & $5 p$ UTR & no & $78 \%$ & $22 \%$ & $0 \%$ \\
\hline miR-150/5127 & chr17:40971032 & 7-mer-m8 & $5 \mathrm{pUTR}$ & no & $78 \%$ & $52 \%$ & $0 \%$ \\
\hline miR-150/5127 & chr17:40971049 & 8-mer & $5 p$ UTR & no & $100 \%$ & $70 \%$ & $0 \%$ \\
\hline miR-15abc/16/16abc/195/322/424/497/1907 & chr17:40966580 & 7-mer-m8 & CDS & no & $100 \%$ & $78 \%$ & $38 \%$ \\
\hline miR-15abc/16/16abc/195/322/424/497/1907 & chr17:40966603 & 7 -mer-m8 & CDS & no & $89 \%$ & $57 \%$ & $0 \%$ \\
\hline miR-17/17-5p/20ab/20b-5p/93/106ab/427/518a-3p/519d & chr17:40962637 & 7-mer-A1 & 3pUTR & no & $100 \%$ & $78 \%$ & $15 \%$ \\
\hline miR-182 & chr17:40971530 & 8-mer & 5pUTR & no & $78 \%$ & $35 \%$ & $0 \%$ \\
\hline miR-187 & chr17:40963690 & 7-mer-m8 & CDS & no & $100 \%$ & $52 \%$ & $15 \%$ \\
\hline miR-187 & chr17:40975808 & 7-mer-A1 & CDS,5pUTR & no & $89 \%$ & $65 \%$ & $0 \%$ \\
\hline miR-199ab-5p & chr17:40962451 & 7-mer-A1 & $3 p U T R$ & no & $100 \%$ & $70 \%$ & $0 \%$ \\
\hline miR-21/590-5p & chr17:40967964 & 7-mer-m8 & CDS & no & $56 \%$ & $4 \%$ & $0 \%$ \\
\hline \begin{tabular}{|l} 
miR-216a \\
\end{tabular} & chr17:40962467 & 7-mer-m8 & 3pUTR & no & $67 \%$ & $48 \%$ & $0 \%$ \\
\hline miR-216a & chr17:40966612 & 8-mer & CDS & no & $100 \%$ & $52 \%$ & $23 \%$ \\
\hline miR-216b/216b-5p & chr17:40962192 & 7-mer-m8 & $3 p U T R$ & no & $100 \%$ & $65 \%$ & $0 \%$ \\
\hline miR-216b/216b-5p & chr17:40966612 & 7-mer-A1 & CDS & no & $100 \%$ & $52 \%$ & $23 \%$ \\
\hline miR-219-5p/508/508-3p/4782-3p & chr17:40966666 & 7-mer-A1 & CDS & no & $78 \%$ & $61 \%$ & $8 \%$ \\
\hline miR-29abcd & chr17:40970981 & 7-mer-m8 & $5 p U T R$ & no & $78 \%$ & $17 \%$ & $0 \%$ \\
\hline miR-30abcdef/30abe-5p/384-5p & chr17:40962673 & 8-mer & $3 p U T R$ & no & $100 \%$ & $91 \%$ & $31 \%$ \\
\hline miR-31 & chr17:40971532 & 7-mer-A1 & $5 p U T R$ & no & $78 \%$ & $43 \%$ & $0 \%$ \\
\hline miR-103a/107/107ab & chr17:40962164 & 7-mer-A1 & $3 p U T R$ & no & $56 \%$ & $30 \%$ & $8 \%$ \\
\hline miR-103a/107/107ab & chr17:40966604 & 7-mer-m8 & CDS & no & $100 \%$ & $78 \%$ & $0 \%$ \\
\hline miR-103a/107/107ab & chr17:40971228 & 7-mer-A1 & $5 p U T R$ & no & $56 \%$ & $0 \%$ & $0 \%$ \\
\hline miR-124/124ab/506 & chr17:40962749 & 7-mer-A1 & 3pUTR & no & $89 \%$ & $57 \%$ & $15 \%$ \\
\hline miR-33ab/33-5p & chr17:40967939 & 7-mer-A1 & CDS & no & $67 \%$ & $43 \%$ & $31 \%$ \\
\hline miR-129-5p/129ab-5p & chr17:40962662 & 7 -mer-A1 & 3pUTR & no & $100 \%$ & $52 \%$ & $15 \%$ \\
\hline
\end{tabular}


Table S2: BCAR4-targeting miRNAs

\begin{tabular}{|c|c|c|c|c|c|c|c|}
\hline \multirow{2}{*}{ microRNA family } & \multirow{2}{*}{ Seed position } & \multirow{2}{*}{ Seed type } & \multirow{2}{*}{ Transcript region } & \multirow{2}{*}{ Repeat } & \multicolumn{3}{|c|}{ Conservation } \\
\hline & & & & & Primates & Mammals & Other vert \\
\hline miR-503 & chr1:94054762 & 8-mer & CDS,ncRNA & no & $89 \%$ & $70 \%$ & $0 \%$ \\
\hline miR-7/7ab & chr1:94033410 & 7 -mer-A1 & ncRNA & no & $78 \%$ & $52 \%$ & $0 \%$ \\
\hline miR-7/7ab & chr1:94054711 & 7-mer-A1 & CDS,ncRNA & no & $89 \%$ & $78 \%$ & $46 \%$ \\
\hline miR-133abc & chr1:94114361 & 7-mer-m8 & ncRNA & no & $67 \%$ & $0 \%$ & $0 \%$ \\
\hline miR-93/93a/105/106a/291a-3p/294/295/302abcde/372/373/428/519a/520be/520acd-3p/1378/1420ac & chr1:94054866 & $7-m e r-m 8$ & CDS,nCRNA & no & $89 \%$ & $74 \%$ & $46 \%$ \\
\hline miR-138/138ab & chr1:94041592 & $7-m e r-m 8$ & $\operatorname{CDS}$ & no & $89 \%$ & $65 \%$ & $8 \%$ \\
\hline miR-146ac/146b-5p & chr1:94057945 & 7-mer-m8 & CDS,nCRNA & no & $89 \%$ & $78 \%$ & $23 \%$ \\
\hline miR-150/5127 & chr1:94079639 & $7-\mathrm{mer}-\mathrm{A} 1$ & $5 p U T R$ & no & $89 \%$ & $43 \%$ & $0 \%$ \\
\hline miR-150/5127 & chr1:94114344 & $7-\mathrm{mer}-\mathrm{A} 1$ & nCRNA & no & $78 \%$ & $0 \%$ & $0 \%$ \\
\hline miR-155 & chr1:94054665 & $7-m e r-m 8$ & CDS,ncRNA & no & $0 \%$ & $0 \%$ & $0 \%$ \\
\hline miR-15abc/16/16abc/195/322/424/497/1907 & chr1:94054762 & $7-\mathrm{mer}-\mathrm{A} 1$ & CDS,ncRNA & no & $89 \%$ & $70 \%$ & $0 \%$ \\
\hline miR-15abc/16/16abc/195/322/424/497/1907 & chr1:94079435 & 7-mer-m8 & CDS & no & $100 \%$ & $83 \%$ & $38 \%$ \\
\hline miR-15abc/16/16abc/195/322/424/497/1907 & chr1:94147375 & 7 -mer-m8 & $5 p U T R$ & no & $67 \%$ & $4 \%$ & $0 \%$ \\
\hline miR-17/17-5p/20ab/20b-5p/93/106ab/427/518a-3p/519d & chr1:94037341 & 7 -mer-m8 & CDS & no & $89 \%$ & $30 \%$ & $0 \%$ \\
\hline let-7/98/4458/4500 & chr1:94311254 & $7-$ mer-A1 & 5pUTR,ncRNA & yes & $44 \%$ & $39 \%$ & $0 \%$ \\
\hline miR-19ab & chr1:94027647 & 7 -mer-A1 & $3 p U T R$ & no & $89 \%$ & $78 \%$ & $31 \%$ \\
\hline miR-1ab/206/613 & chr1:94188935 & 7-mer-A1 & ncRNA & no & $78 \%$ & $0 \%$ & $0 \%$ \\
\hline miR-203 & chr1:94027429 & 7 -mer-m8 & $3 p U T R$ & no & $78 \%$ & $52 \%$ & $15 \%$ \\
\hline miR-203 & chr1:94027927 & 7-mer-A1 & CDS,ncRNA & no & $89 \%$ & $48 \%$ & $23 \%$ \\
\hline miR-204/204b/211 & chr1:94054619 & 7 -mer-A1 & CDS & no & $0 \%$ & $0 \%$ & $0 \%$ \\
\hline miR-205/205ab & chr1:94032957 & 7-mer-A1 & CDS,ncRNA & no & $100 \%$ & $65 \%$ & $0 \%$ \\
\hline miR-205/205ab & chr1:94033301 & 7 -mer-m8 & CDS,ncRNA & no & $78 \%$ & $39 \%$ & $46 \%$ \\
\hline miR-205/205ab & chr1:94037285 & 7 -mer-A1 & CDS & no & $78 \%$ & $26 \%$ & $69 \%$ \\
\hline miR-214/761/3619-5p & chr1:94033004 & 7-mer-A1 & CDS,ncRNA & no & $78 \%$ & $74 \%$ & $31 \%$ \\
\hline miR-214/761/3619-5p & chr1:94147377 & $7-m e r-m 8$ & $5 p U T R$ & no & $67 \%$ & $4 \%$ & $0 \%$ \\
\hline \begin{tabular}{|l|} 
miR-216a \\
\end{tabular} & chr1:94055908 & 7-mer-A1 & ncRNA & yes & $89 \%$ & $17 \%$ & $0 \%$ \\
\hline miR-216b/216b-5p & chr1:94055908 & 7-mer-A1 & ncRNA & yes & $89 \%$ & $17 \%$ & $0 \%$ \\
\hline miR-217 & chr1:94054813 & 7-mer-A1 & CDS,ncRNA & no & $67 \%$ & $74 \%$ & $0 \%$ \\
\hline miR-217 & chr1:94079469 & 7 -mer-m8 & CDS & no & $56 \%$ & $22 \%$ & $0 \%$ \\
\hline miR-22/22-3p & chr1:94048067 & $7-m e r-m 8$ & CDS,ncRNA & no & $44 \%$ & $0 \%$ & $0 \%$ \\
\hline miR-22/22-3p & chr1:94188993 & 7-mer-A1 & ncRNA & no & $33 \%$ & $0 \%$ & $0 \%$ \\
\hline miR-122/122a/1352 & chr1:94140258 & 7-mer-A1 & CDS & no & $67 \%$ & $26 \%$ & $0 \%$ \\
\hline miR-23abc/23b-3p & chr1:94107861 & 7-mer-A1 & CDS,ncRNA & no & $89 \%$ & $74 \%$ & $23 \%$ \\
\hline miR-24/24ab/24-3p & chr1:94048417 & 7 -mer-m8 & CDS,ncRNA & no & $100 \%$ & $52 \%$ & $31 \%$ \\
\hline miR-24/24ab/24-3p & chr1:94054591 & 7 -mer-m8 & CDS & no & $56 \%$ & $61 \%$ & $31 \%$ \\
\hline miR-24/24ab/24-3p & chr1:94055876 & 8-mer & ncRNA & yes & $89 \%$ & $4 \%$ & $0 \%$ \\
\hline miR-24/24ab/24-3p & chr1:94055919 & $7-$ mer-A1 & ncRNA & yes & $56 \%$ & $43 \%$ & $0 \%$ \\
\hline miR-25/32/92abc/363/363-3p/367 & chr1:94108742 & $7-m e r-m 8$ & nCRNA & yes & $56 \%$ & $0 \%$ & $0 \%$ \\
\hline miR-26ab/1297/4465 & chr1:94048114 & $7-m e r-m 8$ & CDS,ncRNA & yes & $67 \%$ & $61 \%$ & $0 \%$ \\
\hline$m i R-27 a b c / 27 a-3 p$ & chr1:94032921 & 7-mer-A1 & CDS,ncRNA & no & $100 \%$ & $35 \%$ & $38 \%$ \\
\hline$m i R-27 a b c / 27 a-3 p$ & chr1:94048251 & 8-mer & CDS,nCRNA & no & $100 \%$ & $70 \%$ & $8 \%$ \\
\hline miR-29abcd & chr1:94050535 & 7 -mer-m8 & 5 pUTR,ncRNA & yes & $89 \%$ & $35 \%$ & $0 \%$ \\
\hline miR-338/338-3p & chr1:94050591 & 7-mer-A1 & 5pUTR,ncRNA & no & $89 \%$ & $43 \%$ & $0 \%$ \\
\hline miR-338/338-3p & chr1:94188910 & $7-m e r-m 8$ & nCRNA & no & $44 \%$ & $4 \%$ & $0 \%$ \\
\hline miR-33a-3p/365/365-3p & chr1:94033487 & 7-mer-A1 & ncRNA & yes & $22 \%$ & $0 \%$ & $0 \%$ \\
\hline miR-33ab/33-5p & chr1:94188872 & 7 -mer-A1 & nCRNA & no & $33 \%$ & $0 \%$ & $0 \%$ \\
\hline$m i R-34 a c / 34 b c-5 p / 449 a b c / 449 c-5 p$ & chr1:94033351 & 7-mer-A1 & CDS, ncRNA & no & $67 \%$ & $13 \%$ & $23 \%$ \\
\hline miR-125a-5p/125b-5p/351/670/4319 & chr1:94107897 & 7 -mer-A1 & CDS & no & $100 \%$ & $74 \%$ & $15 \%$ \\
\hline miR-10abc/10a-5p & chr1:94033567 & $7-m e r-m 8$ & ncRNA & yes & $67 \%$ & $0 \%$ & $0 \%$ \\
\hline miR-455-5p & chr1:94057930 & 7-mer-m8 & CDS,ncRNA & no & $67 \%$ & $22 \%$ & $0 \%$ \\
\hline miR-455-5p & chr1:94311170 & $7-m e r-m 8$ & 5pUTR,ncRNA & yes & $44 \%$ & $0 \%$ & $0 \%$ \\
\hline miR-128/128ab & chr1:94048252 & $7-\mathrm{mer}-\mathrm{A} 1$ & CDS, ncRNA & no & $100 \%$ & $70 \%$ & $8 \%$ \\
\hline miR-490-3p & chr1:94033662 & 7 -mer-m8 & ncRNA & yes & $22 \%$ & $0 \%$ & $0 \%$ \\
\hline
\end{tabular}

